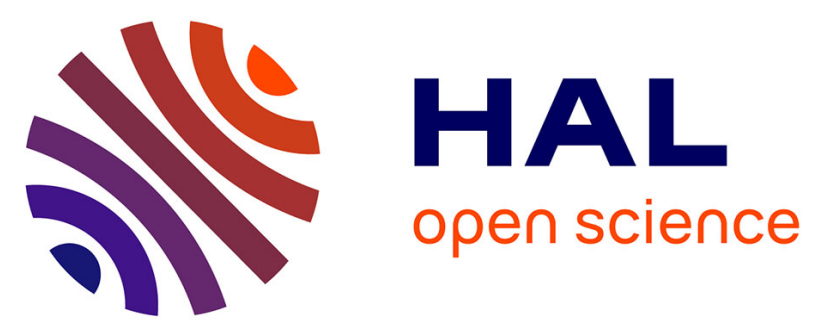

\title{
Nature of the Polyanion Governs the Antimicrobial Properties of Poly(arginine)/Polyanion Multilayer Films
}

Angela Mutschler, Cosette Betscha, Vincent Ball, Bernard Senger, Nihal Engin Vrana, Fouzia Boulmedais, André Schroder, Pierre Schaaf, Philippe Lavalle

\section{To cite this version:}

Angela Mutschler, Cosette Betscha, Vincent Ball, Bernard Senger, Nihal Engin Vrana, et al.. Nature of the Polyanion Governs the Antimicrobial Properties of Poly(arginine)/Polyanion Multilayer Films. Chemistry of Materials, 2017, 29 (7), pp.3195-3201. 10.1021/acs.chemmater.7b00334 . hal-02142321

\section{HAL Id: hal-02142321 \\ https://hal.science/hal-02142321}

Submitted on 5 Jan 2021

HAL is a multi-disciplinary open access archive for the deposit and dissemination of scientific research documents, whether they are published or not. The documents may come from teaching and research institutions in France or abroad, or from public or private research centers.
L'archive ouverte pluridisciplinaire HAL, est destinée au dépôt et à la diffusion de documents scientifiques de niveau recherche, publiés ou non, émanant des établissements d'enseignement et de recherche français ou étrangers, des laboratoires publics ou privés. 


\title{
Nature of the Polyanion Governs the Antimicrobial Properties of Poly(arginine)/Polyanion Multilayer Films
}

\author{
Angela Mutschler, ${ }^{\dagger, \uparrow}$ Cosette Betscha, ${ }^{\dagger \dagger}$ Vincent Ball, ${ }^{\dagger, \ddagger}$ Bernard Senger, ${ }^{\dagger \dagger}$ Nihal Engin Vrana, ${ }^{\dagger, \S_{0}}$ \\ Fouzia Boulmedais, ${ }^{\| \odot}$ André Schroder, $\|$ Pierre Schaaf, $, \dagger,+, \|$ and Philippe Lavalle ${ }^{*, \dagger, \ddagger(0)}$ \\ ${ }^{\dagger}$ INSERM, UMR 1121, 11 Rue Humann, 67085 Strasbourg, France \\ ${ }^{\ddagger}$ Fédération de Médecine Translationnelle de Strasbourg, Fédération Matériaux et Nanosciences d'Alsace, Faculté de Chirurgie \\ Dentaire, Université de Strasbourg, 67000 Strasbourg, France \\ ${ }^{\S}$ ProtipMedical, 8 Place de l'Hôpital, 67000 Strasbourg, France \\ "CNRS Institut Charles Sadron UPR 22, Université de Strasbourg, 23 Rue du Lœss, 67034 Strasbourg, France
}

Supporting Information

\begin{abstract}
Coatings with antimicrobial properties are garnering interest to prevent implant-associated infections. Recently, we showed that poly(arginine)/hyaluronic acid (PAR/HA) multilayers built with PAR chains constituted from 30 arginine residues (PAR30) have strong antimicrobial properties through a contact-killing mechanism. This property is due to the ability of PAR30 chains, when associated with HA, to diffuse in the multilayer. Here, we investigate the effect of the nature of the polyanion on the antimicrobial activity of (PAR30/polyanion) multilayers. Four polysaccharides, one polypeptide, and one synthetic polyelectrolyte are investigated. Surprisingly, only HA leads to films with antimicrobial character. We relate this property to the strong diffusion capacity of PAR30 chains in (PAR30/HA) multilayers compared to their diffusion ability in the other (PAR30/polyanion) films. Through isothermal microcalorimetry experiments, we also demonstrate that interactions in solutions of PAR30 chains with the different polyanions are characterized by a negative reaction enthalpy for all of the investigated polyanions except for HA, where the enthalpy of reaction is positive. Moreover, the molecular weight of HA is not a key parameter for the diffusion ability of PAR chains or for the bioactivity of the film. These results constitute an important step toward the establishment of rules to design contact-killing antimicrobial polyelectrolyte multilayers.

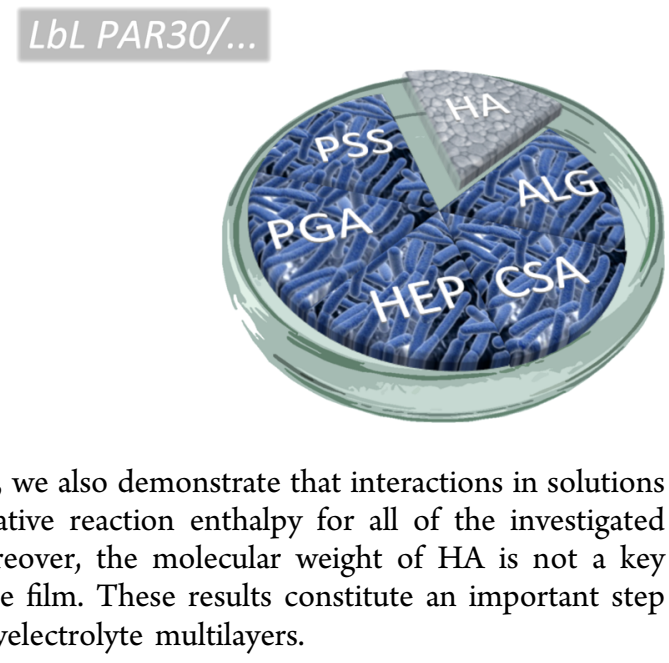

\section{INTRODUCTION}

Infections represent one of the major risks accompanying implant surgery. ${ }^{1,2}$ In parallel, the rise of resistance of some bacteria strains to antibiotics renders their treatment increasingly difficult and constitutes a major medical threat in the future. ${ }^{3,4}$ These observations stimulate the development of antimicrobial coatings aimed to prevent implant infections during the first hours or days after implantation. ${ }^{5}$ Among the different methods that are under investigation to confer an antimicrobial character to a material, polyelectrolyte multilayers have received great attention. ${ }^{6}$ Obtained by the alternate deposition of polyanions and polycations, they allow functionalizing almost any kind of surface and their applications range from optical coating and superhydrophobic and conductive surfaces up to biomaterial functionalization. ${ }^{7,8}$ Polyelectrolyte multilayers allow designing, in particular, antimicrobial surfaces. ${ }^{9,10}$ Three strategies are reported to achieve antimicrobial polyelectrolyte multilayers: ${ }^{11}(\mathrm{i})$ multilayers that prevent bacterial adhesion, ${ }^{12}$ (ii) multilayers that kill bacteria through direct contact, ${ }^{13}$ and (iii) multilayers that release antimicrobial agents. ${ }^{14-16}$

Recently, we found that poly(arginine)/hyaluronic acid (PAR/HA) multilayers present interesting biological behav- iors. ${ }^{17}$ They show some immunomodulatory properties, and in addition they present a strong antimicrobial character against Staphylococcus aureus (S. aureus). More interestingly, this latter property is strongly dependent upon the molecular weight of PAR. ${ }^{18}$ Whereas multilayers constituted from 24 PAR/HA bilayers built with PAR of 10, 100, or 200 residues show almost no effect against $S$. aureus, films built with PAR chains with 30 residues display strong antimicrobial properties. This constitutes, to our knowledge, the first example of a multilayer whose functional property is dependent upon the size of the polyelectrolyte chains used for the film buildup. PAR/HA is a multilayer whose thickness increases exponentially with the number of deposition steps (at least during the first 1015 bilayer deposition steps). This growth mechanism is related to the ability of at least one of the polyelectrolytes constituting the film to diffuse in and out of the whole film during each deposition step. ${ }^{19}$ For PAR30/HA multilayers, PAR30 chains are the diffusing species and this diffusion ability is at the origin 
Scheme 1. Chemical Structures of the Polyanions Used in the Present Study: ALG (a), CSA (b), HA (c), HEP (d), PGA (e), and PSS $(\mathbf{f})^{a}$

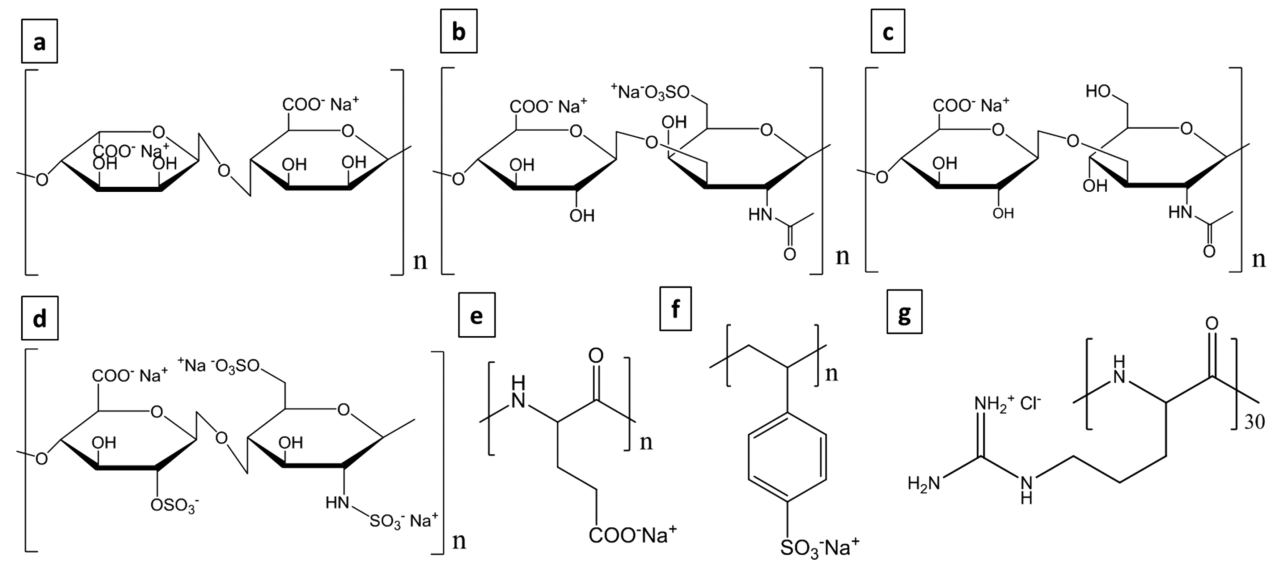

${ }^{a_{T}}$ These polyanions are associated with the polycation $\operatorname{PAR}(\mathrm{g})$.

of the contact-killing property of the film. When bacteria adhere to the film, the positively charged PAR chains diffuse from the film toward the negatively charged bacterial membranes and destabilize them. The difference in antimicrobial efficiency of PAR/HA films is explained by the smaller diffusion ability (smaller proportion of mobile chains) of longer PAR chains (above 100 residues) compared to a smaller one (30 residues). For films built with PAR chains of 10 residues, it appears that with 24 bilayers the number of PAR chains is not sufficient to kill all the bacteria after $24 \mathrm{~h}$.

With the idea to develop a new family of antimicrobial films and to establish rules to design contact-killing polyelectrolyte multilayer films, here we investigate the effect of the nature of different polyanions on the antimicrobial activity of the PAR30/polyanion multilayers. In other words, is hyaluronic acid essential for antimicrobial properties of PAR-based films? HA will be compared to three polysaccharides, alginate (ALG), heparin (HEP), and chondroitin sulfate A (CSA), to a homopolypeptide, poly(L-glutamic acid) (PGA), and to a synthetic polyelectrolyte, poly(sodium 4-styrene sulfonate), one of the most investigated polyanions in the multilayer literature. The chemical structures of the different polyanions are given in Scheme 1. PAR chains with 30 monomers will be used as polycations along this study (PAR30).

\section{EXPERIMENTAL SECTION}

Materials. The polyelectrolyte multilayer films have been built up with the following polymers. The polycation was poly(L-arginine hydrochloride) whose chains consisted of 30 residues (PAR30, 30R; $\mathrm{MW}=6.4 \mathrm{kDa}$, PDI $=1.01)$ and was purchased from Alamanda Polymers, USA. Hyaluronic acid (HA, MW $=29$ and $150 \mathrm{kDa})$ used as a polyanion was produced by Lifecore Biomed, USA. Alginic acid was purified from brown algae (ALG; MW $=240 \mathrm{kDa}$, Sigma, France), chondroitin sulfate A from bovine trachea (CSA; Sigma, France), and heparin sodium salt from porcine intestinal mucosa (HEP; MW = $18 \mathrm{kDa}$, Sigma, France). Poly(L-glutamic acid) (PGA) and poly(styrene sodium sulfonate) (PSS; $\mathrm{MW}=70 \mathrm{kDa}$ ) used as the polyanions were purchased from Sigma. Tris(hydroxymethyl)aminomethane (TRIS) was purchased from Merck, Germany.

Monitoring the Buildup of Films. Quartz crystal microbalance with dissipation monitoring (QCM-D, E1, Q-Sense, Sweden) was used to monitor in situ film buildup. The quartz crystal was excited at its fundamental frequency (about $5 \mathrm{MHz}$ ), as well as at the third, fifth, seventh, and ninth overtones (denoted by $\nu=3,5,7$, and 9 and corresponding respectively to $15,25,35$, and $45 \mathrm{MHz}$ ). Changes in the resonance frequencies $\left(-\Delta f_{\nu}\right)$ were measured at these four frequencies. An increase of the normalized frequency $-\Delta f_{\nu} / \nu$ is often associated with a proportional increase of the mass coupled with the quartz crystal (Sauerbrey's approximation). ${ }^{20}$ However, when the normalized frequency shifts for the various overtones are unequal, it is advisible to use the model developed by Voinova et al., ${ }^{21}$ where both the measured frequency shifts and dissipations for several overtones are processed to derive the thickness. This model relies on the hypothesis that the film is a homogeneous, isotropic, and nonsliding viscoelastic stratum deposited on the quartz crystal. Data corresponding to the overtones $\nu=3,5,7$, and 9 have been used to determine the thicknesses of all films used here.

PAR and polyanions were dissolved at $0.5 \mathrm{mg} \cdot \mathrm{mL}^{-1}$ in sterilized buffer containing $150 \mathrm{mM} \mathrm{NaCl}$ and $10 \mathrm{mM}$ TRIS at pH 7.4. Polycationic and polyanionic solutions were successively injected into the QCM-D cell containing the $\mathrm{SiO}_{2}$-coated quartz crystal. PAR30 was the first deposited polyelectrolyte. Each polyelectrolyte was adsorbed for $5 \mathrm{~min}$, and then a rinsing step with NaCl-TRIS buffer was performed for $5 \mathrm{~min}$.

Buildup of (PAR/HA) ${ }_{24}$ Films. For the construction of 24 bilayers of PAR30/polyanion (denoted as (PAR30/polyanion) $)_{24}$ ), an automated dipping robot was used (Riegler \& Kirstein GmbH, Germany). Glass slides (12 mm in diameter) were first washed with Hellmanex II solution at $2 \%, 1 \mathrm{M} \mathrm{HCl}, \mathrm{H}_{2} \mathrm{O}$, and ethanol and dried with an air flow. Solutions of polyelectrolytes were prepared as described above for QCM-D experiments. Glass slides were dipped alternately in the polycation and polyanion solutions and extensively rinsed in $\mathrm{NaCl}-$ TRIS buffer between each step. After construction, the films were dried with an air flow, then immersed in NaCl-TRIS buffer, and stored at $4{ }^{\circ} \mathrm{C}$ before use. Observations of the films were carried out with a confocal Zeiss LSM 710 microscope (Heidelberg, Germany) using a $20 \times$ Plan Apo (0.8 NA) objective (Zeiss). The microscopic observations of the obtained films were evaluated by deposition of $100 \mu \mathrm{L}$ of PAR-FITC (PAR labeled with fluorescein isothiocyanate, $0.5 \mathrm{mg} \cdot \mathrm{mL}^{-1}$ in NaCl-TRIS buffer) on top of the PAR30/polyanion multilayer films. Fluorescent labeling of PAR was previously described. ${ }^{18}$ After 5 min of deposition and diffusion of PAR-FITC through the whole film, a rinsing step was performed with NaCl-TRIS buffer.

Antibacterial Assay. S. aureus (ATCC 25923) strain was used to assess the antibacterial properties of the samples. The bacterial strain was cultured aerobically at $37{ }^{\circ} \mathrm{C}$ in a Mueller Hinton Broth (MHB) medium (Merck, Germany), pH 7.4. One colony was transferred to $10 \mathrm{~mL}$ of $\mathrm{MHB}$ medium and incubated at $37^{\circ} \mathrm{C}$ for $20 \mathrm{~h}$. To obtain bacteria in the mid-logarithmic phase of growth, the absorbance at $620 \mathrm{~nm}$ of overnight culture was adjusted to 0.001 , corresponding to a final density of $8 \times 10^{5} \mathrm{CFU} \cdot \mathrm{mL}^{-1}$.

Glass slides coated with (PAR30/polyanion) ${ }_{24}$ films were sterilized by using UV light during $15 \mathrm{~min}$ and then washed with NaCl-TRIS 
buffer. After washing, all glass slides were deposited in 24-well plates with $300 \mu \mathrm{L}$ of $S$. aureus, $A_{620}=0.001$, and incubated during $24 \mathrm{~h}$ at $37{ }^{\circ} \mathrm{C}$.

For negative control, uncoated glass slides were directly incubated with $S$. aureus using a similar method. To quantify bacteria growth or inhibition after $24 \mathrm{~h}$, the absorbance of the supernatant at $620 \mathrm{~nm}$ was measured.

Bacteria Live Dead Assay. The BacLight RedoxSensor CTC Vitality Kit (Thermo Fisher Scientific Inc., France) was used for evaluation of the health of bacteria present on the surface. This kit gives a semiquantitative estimate of healthy vs unhealthy bacteria. SYTO 24 green-fluorescent nucleic acid stain (Thermo Fisher Scientific Inc., France) was used for counting all bacteria. A solution of $50 \mathrm{mM} \mathrm{CTC}$ and $1 \mu \mathrm{M}$ SYTO 24 in pure water was prepared. All glass slides were washed with phosphate-buffered saline buffer (PBS), $\mathrm{pH}=7.4$. Then $270 \mu \mathrm{L}$ of PBS and $30 \mu \mathrm{L}$ of CTC/SYTO 24 solution were added. The plates were incubated for $30 \mathrm{~min}$ at $37^{\circ} \mathrm{C}$, away from light. Each surface was observed by confocal microscopy (Zeiss LSM 710 microscope, Heidelberg, Germany), using $63 \times$ Plan Apo (1.4 NA) objective immersed in oil. Excitation/emission wavelengths of stains were $450 \mathrm{~nm} / 630 \mathrm{~nm}$ for CTC and $490 \mathrm{~nm} / 515 \mathrm{~nm}$ for SYTO 24 .

Fluorescence Recovery after Photobleaching Experiments. The proportion of mobile molecules, $p$, was measured for (PAR30/ polyanion $)_{24}$ films containing PAR30-FITC by performing fluorescence recovery after photobleaching (FRAP) experiments. A glass slide coated with a PAR30/polyanion film was introduced in a homemade sample holder and immersed in $200 \mu \mathrm{L}$ of NaCl-TRIS buffer. One circular region $(4.4 \mu \mathrm{m}$ in radius in an image of $35 \mu \mathrm{m} \times 35 \mu \mathrm{m})$ was exposed for $700 \mathrm{~ms}$ to the light of a laser set at its maximum power $(\lambda=488 \mathrm{~nm})$. Then, the recovery of fluorescence in the bleached area was followed over time. Observations were carried out with a Zeiss LSM 710 microscope (Heidelberg, Germany) using a 20X Plan Apo (0.8 NA) objective (Zeiss). At the same time, three equally sized circular reference areas outside of the bleached area were monitored. The intensities in these areas are used to normalize the intensity in the bleached area so that bleaching due to image acquisition was accounted for.

Under the hypothesis that (i) the recovery is due to the Brownian diffusion of the mobile labeled molecules, (ii) all moving labeled molecules have the same diffusion coefficient, and (iii) the initial intensity profile (i.e., immediately after the bleaching) has a sharp edge

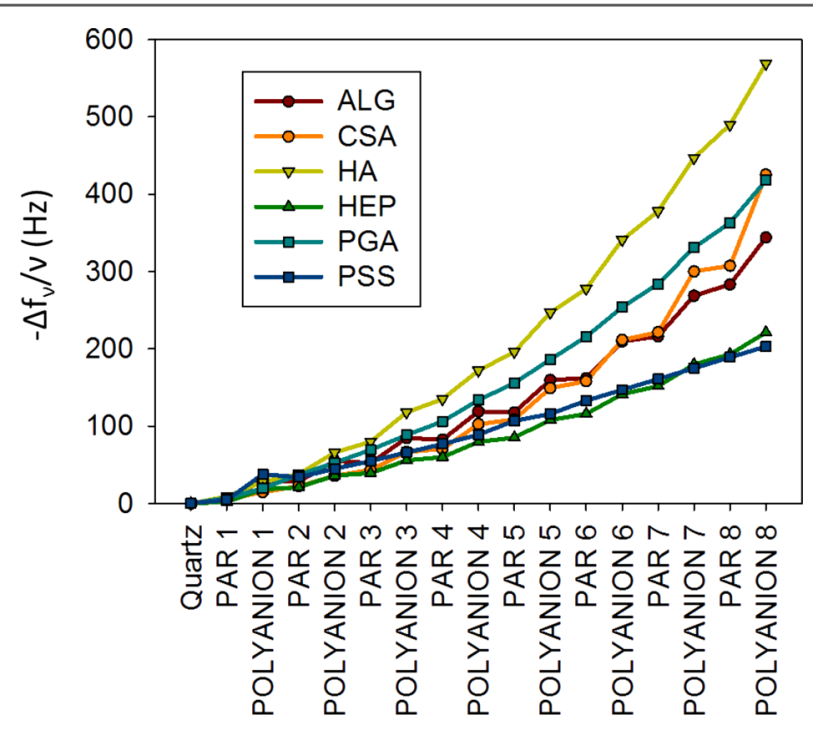

Figure 1. Buildup of (PAR30/polyanion $)_{8}$ multilayer film on a $\mathrm{SiO}_{2}$ coated crystal followed by QCM-D with ALG (dark red), CSA (orange), HA (light green), HEP (dark green), PGA (light blue), and PSS (dark blue) as polyanions. The evolution of the normalized frequency $-\Delta f_{\nu} / \nu($ for $\nu=3)$ as a function of the number of adsorbed layers is shown. (a circle in the present case), the time evolution of the normalized intensity can be derived theoretically. ${ }^{22,23}$ It depends on the diffusion coefficient, $D$, and the proportion of mobile labeled molecules, $p$, as well as the radius, $R$, of the initially bleached area. Note that, according to the aforementioned theory, the area observed during the recovery must have the same radius and the same center as the bleached area. Bleached areas were observed for at least $6 \mathrm{~min}$.

Isothermal Titration Calorimetry. We used a Nano isothermal titration calorimetry (ITC) standard volume microcalorimeter (TA Instruments, USA) to measure interaction isotherms during the titration of the polyanions solutions with PAR30. Experiments were performed at $25^{\circ} \mathrm{C}$. The microcalorimeter measurement and reference cell volumes were $1 \mathrm{~mL}$. The reference cell contained Milli- $\mathrm{Q}$ water, and the sample cell contained the polyanions solution. PAR30 and polyanions were separately dissolved in $\mathrm{NaCl}$-TRIS buffer solutions with concentrations of $5.16 \mathrm{mM}$ and $0.516 \mathrm{mM}$ of residue, respectively. PAR30 was placed in a $250 \mu \mathrm{L}$ motor-driven syringe (titrant), each titration experiment consisting of a series of 25 injections of $10 \mu \mathrm{L}$, with a waiting time of $600 \mathrm{~s}$ between two successive injections, at a constant stirring rate of $300 \mathrm{rpm}$. As a consequence, the arginine/ anionic residues ratio reached the final value of 2.5 at the end of a titration experiment. The heat associated with the dilution of PAR30

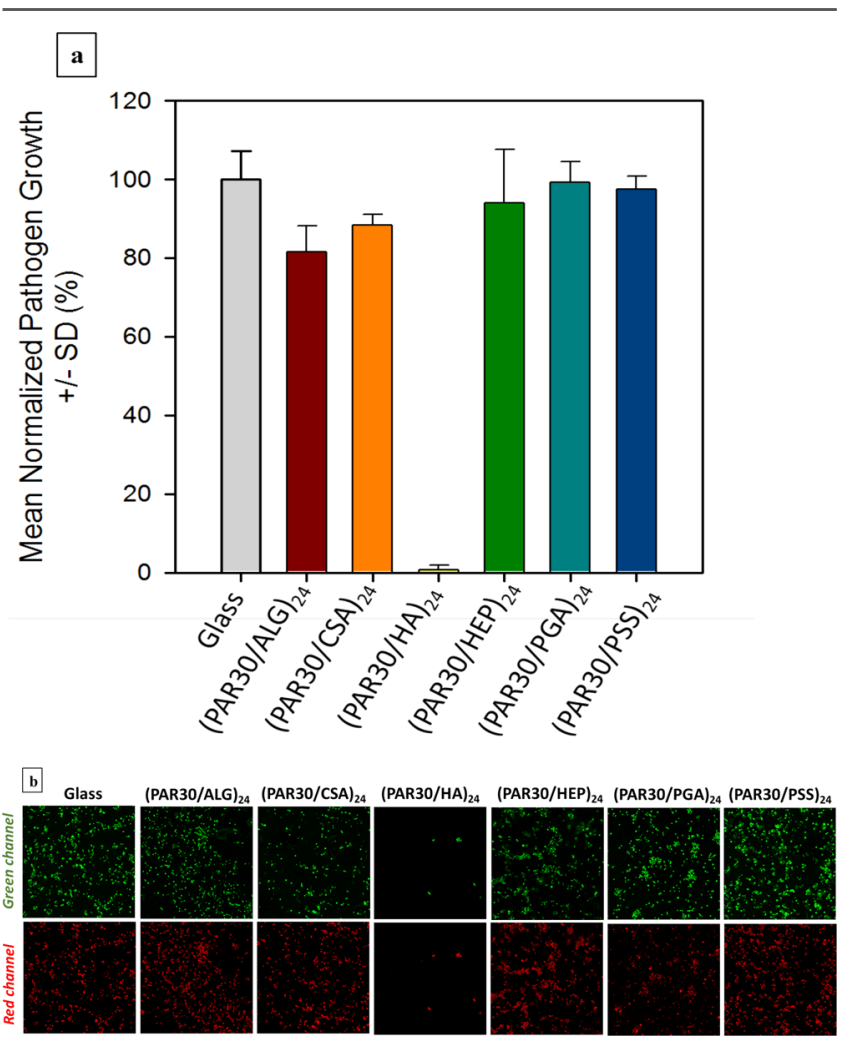

Figure 2. (a) Normalized $S$. aureus growth in the supernatant after $24 \mathrm{~h}$ in contact with (PAR30/polyanion) $)_{24}$ multilayer films built with ALG, CSA, HEP, HA, PGA, and PSS as polyanions. The height of the colored bar represents the mean value of several independent measurements, and the error bars correspond to standard deviations (SDs). Sample sizes: $n=15$ for glass and $n=3$ for PAR30/polyanions. The pathogen growth has been arbitrarily normalized to $100 \%$ for glass (gray bar), and the other measurements have been normalized accordingly. (b) Confocal images of $S$. aureus after $24 \mathrm{~h}$ of incubation and on glass substrates, on (PAR/polyanion) ${ }_{24}$ films. Images in the green channel correspond to SYTO 24 labeling for counting all bacteria and images in the red channel correspond to healthy bacteria through metabolism of CTC (5-cyano- 2,3-ditolyltetrazolium chloride) into an insoluble red-fluorescent formazan. Image sizes are $67 \times 67 \mu \mathrm{m}^{2}$. 


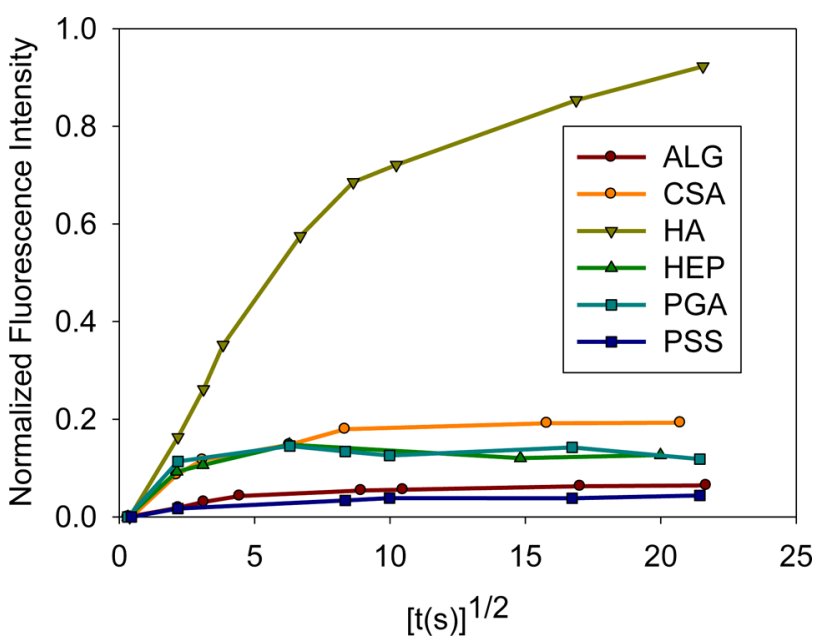

Figure 3. Normalized fluorescence intensity of a photobleached area according to $[t]^{1 / 2}$ for each film: (PAR30-FITC/polyanion) ${ }_{24}$, with ALG (dark red), CSA (orange), HA (light green), HEP (dark green), PGA (light blue), and PSS (dark blue) as polyanions. $t=0$ corresponds to the end of the photobleaching step.

in $\mathrm{NaCl}$-TRIS buffer was measured with the same conditions, leading to an average value of $-20 \mu \mathrm{J}$ per injection. This contribution was subtracted from the surface area of the peaks in the thermograms before calculating the molar heat associated with the interaction of PAR30 with a polyanion.

Statistical Analysis. The data concerning evaluation of pathogen growth were processed by using SigmaPlot (Systat Software Inc., USA). The one-way ANOVA test was performed at a significance level $\alpha=0.05$.

\section{RESULTS AND DISCUSSION}

We first investigated the growth of the different multilayers by quartz crystal microbalance with dissipation monitoring (QCM-D). Figure 1 represents the evolution of the frequency shift of the third overtone $(15 \mathrm{MHz})$ during the first eight deposition steps. After eight deposited pairs of layers (or "bilayers") the thicknesses of the films built with PAR30 and HA, PGA, ALG, CSA, HEP, and PSS are equal to 120, 95, 72, 68, 42 , and $40 \mathrm{~nm}$, respectively (Supporting Information Figure S1). Between all combinations, PAR30/HA has the strongest exponential behavior. Among the different investigated polysaccharides, HEP is the most negatively charged (one sulfonate group, two sulfate groups, and one carboxylate group per monomer), CSA and ALG are intermediate (one carboxylate and one sulfate group per CSA monomer and two carboxylate groups per ALG monomer), whereas HA is the least negatively charged polysaccharide (one carboxylate group per monomer) (Scheme 1). As can be observed in Figures 1 and S1, the exponential character decreases in the order HA $>$ ALG $\sim$ CSA $>$ HEP while the negative charge on the chains increases in the order $\mathrm{HA}<\mathrm{ALG} \sim \mathrm{CSA}<\mathrm{HEP}$. Moreover it was previously shown that due to the kosmotropic character of $\mathrm{COO}^{-}$and chaotropic character of $\mathrm{SO}_{3}{ }^{-}$, films formed with $\mathrm{HA}$ are more hydrated than films formed with HEP or CSA. ${ }^{24}$ Not only do (PAR/HA) films seem to be the most hydrated ones but they also have the weakest electrostatic interactions. This point will be discussed later. This result strengthens the conclusion that the exponential character of the films increases when the polycation/polyanion interaction decreases. ${ }^{24}$

Next we investigated the homogeneity of the films. PAR labeled with FITC (PAR-FITC) and confocal microscopy were used for this purpose. Along the $(x, z)$ plane, all films appeared very homogeneous and a uniform coating on the surface was obtained as stated by the green homogeneous bands observed in Figure S2. This property was not guaranteed since some polysaccharide/polyanion multilayers form some droplet-like structures whose origin has been the subject of previous study. ${ }^{25}$ Yet, the homogeneity of the films is important to expect antimicrobial properties of the functionalized surfaces.

We then investigated the antimicrobial property of these PAR based multilayers against $S$. aureus, which is at the origin of more than $50 \%$ of the infections associated with implantable devices. ${ }^{26}$ Two types of experiments were performed. A solution of $S$. aureus in $\mathrm{MH}$ medium was brought in contact with the polyelectrolyte multilayer under stirring, and the pathogen growth in the solution was determined after $24 \mathrm{~h}$ of contact by measuring the absorbance at $620 \mathrm{~nm}$ of the supernatant. In the second method, we evaluated the presence of the bacteria deposited on the substrate after $24 \mathrm{~h}$ of contact by microscopy. When using PAR30 chains, only (PAR30/HA) ${ }_{24}$ multilayers showed a quasi-total inhibition of bacterial growth in solution (Figure 2a). Moreover, using the live/dead test and microscopic observations, it was hard to visualize an area with the presence of bacteria on this surface (Figure 2b). For all other multilayers, the normalized $S$. aureus growth in solution laid between 81 and $97 \%$ of the control (noncoated surface, 100\%). By statistical analysis (one-way ANOVA test), we confirmed a significant difference between (PAR30/HA $)_{24}$ and glass, as well as $(\mathrm{PAR} 30 / \mathrm{HA})_{24}$ and the other multilayers. The same test revealed also a significant difference between (PAR30/ALG) ${ }_{24}$ and glass. However, inhibition of bacterial growth is weak for (PAR30/ALG) ${ }_{24}$, and this is confirmed by microscopic observations. The other multilayers showed neither mutual statistical differences in the pathogen growth in solution nor a difference with glass. Finally, we observe a significant growth inhibition of S. aureus on PAR30/HA films in contrast to other multilayers.

The activity of the film could not be explained by a bactericidal effect of the polyanionic chains because inhibition of the bacterial growth up to $50 \mu \mathrm{M}$ (Figure S3) was very limited when polyanions were incubated in solution with bacteria. We observed that none of the ALG, CSA, HA, HEP, PGA, or PSS chains show strong antimicrobial properties, PSS

Table 1. Reaction Enthalpies for the Investigated PAR30/Polyanion Couple

$\begin{array}{ccccc}\text { polyanion } & \text { negative charge/residue } & \text { growth regime of the film } & \text { antimicrobial properties of the (PAR30/polyanion) film } & - \\ \text { ALG } & 1 & \text { exponential } & - & -3.81 \\ \text { CSA } & 1 & \text { exponential } & + \\ \text { HA } & 0.5 & \text { exponential } & -3.90 \\ \text { HEP } & 2 & \text { weakly exponential } & - \\ \text { PGA } & 1 & \text { exponential } & - \\ \text { PSS } & 1 & \text { linear } & -\end{array}$


being the more effective at $50 \mu \mathrm{M}$ but with a reduction of proliferation limited to $70 \%$. Comparatively, PAR chains at $50 \mu \mathrm{M}$ show $100 \%$ inhibition and, at $10 \mu \mathrm{M}$, inhibition is close to $90 \%$.

FRAP experiments were performed in order to determine the diffusion ability of the PAR30 chains in the different films. Figure 3 represents the evolution of the recovered fluorescence of the bleached zone for each couple (PAR30-FITC/polyanion). Striking differences exist between the different polyanions over the FRAP experimental time scale (about $20 \mathrm{~min}$ ). Whereas up to $90 \%$ of the PAR30 chains diffuse in PAR/HA films, only $20 \%$ diffuse in CSA based films, of the order of $10 \%$ diffuse in HEP and PGA-based films and of the order of 5\% in ALG-based and PGA-based films. These results show that the antimicrobial character of the PAR/polyanion multilayers is probably directly related to the ability of the PAR chains to diffuse in the film. In particular, this provides an explanation of the antimicrobial effect observed only with HA. The diffusion ability of the PAR chains thus appears to be extremely sensitive not only to the PAR chain length but also to the nature of the associated polyanion. These results show how by knowing the basic physicochemical rules governing a multilayer film behavior, one can predict and finely tune its properties for functionalization purposes.

The stability of the (PAR30-FITC/HA) 24 and (PAR30-FITC/ ALG) ${ }_{24}$ multilayers (ALG is used as control) was also tested through observations with confocal microscopy after incubation at $37{ }^{\circ} \mathrm{C}$ during $24 \mathrm{~h}$ in contact with $\mathrm{MH}$ medium with or without S. aureus (Figure S4). Images of (PAR30-FITC/HA) 24 show vermiculate patterns with "islands" after $24 \mathrm{~h}$ of incubation with or without bacteria. However, as demonstrated previously, practically no release of the PAR takes place during this process and no degradation of the film was monitored. ${ }^{18}$ These patterns could be due to a rearrangement of the film and a dewetting process induced by the $\mathrm{MH}$ medium but not to a degradation process. Moreover, bacteria seem not to be responsible for this rearangement. For (PAR30-FITC/ALG) ${ }_{24}$ control films, after $24 \mathrm{~h}$ of incubation in $\mathrm{MH}$ medium with or without $S$. aureus the multilayer remains homogeneous and no patterns are observed. As demonstrated before, most of the ALG chains are not mobile in the film. This suggests that probably the high mobility of PAR30 chains is at the origin of the vermiculate pattern observed with PAR30/HA films during incubation in $\mathrm{MH}$ medium.

To better understand the mechanism responsible for PAR diffusion, we determined the heat of complexation for each couple (PAR30/polyanion). Previous studies showed that an endothermic complexation process between polycations and polyanions is characteristic of an exponential film growth, whereas a strongly exothermic process corresponds to a linear growth regime. ${ }^{27}$ Thus, the strength of the polycation/polyanion interaction, measured by the heat of complexation, could be related to the polyelectrolyte diffusion ability in the films, and finally a direct relation could be established with the antimicrobial character of the corresponding film. In order to validate this hypothesis, we used isothermal titration microcalorimetry (ITC) to determine the enthalpy of the polycation/ polyanion complexation in solution (Figure S5). Table 1 represents the reaction enthalpy measured for each couple of (PAR30/polyanion).

One clearly observes that among the six investigated systems, the interaction of PAR with HA is the only one that is endothermic, indicating a weak interaction. The PAR/HA film buildup must be driven by an entropic process. After buildup, the diffusion ability of the PAR chains should no longer depend
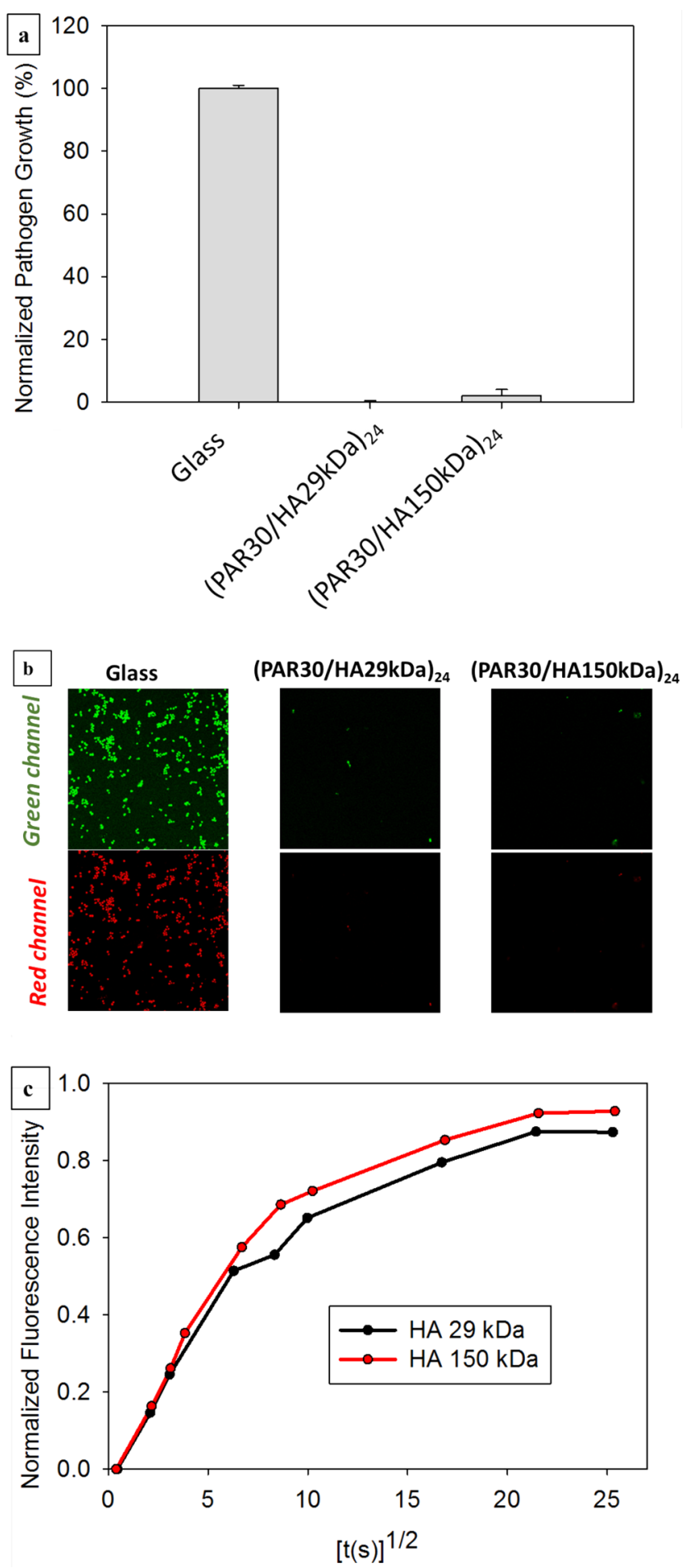

Figure 4. (a) Normalized $S$. aureus growth in the supernatant after $24 \mathrm{~h}$ in contact with (PAR30/HA) 24 multilayers built with HA of molecular weight of 29 or $150 \mathrm{kDa}$. Each value corresponds to the mean value of three individual experiments, and error bars correspond to standard deviations. (b) Confocal images of $S$. aureus after $24 \mathrm{~h}$ of incubation on glass substrates and on (PAR30/HA) 24 films. Images in the green channel correspond to SYTO 24 labeling for counting all bacteria, and images in the red channel correspond to healthy bacteria through metabolism of CTC (5-cyano- 2,3-ditolyltetrazolium chloride) into an insoluble red-fluorescent formazan. Image sizes are $67 \times 67 \mu \mathrm{m}^{2}$. (c) Normalized fluorescence intensity of a photobleached area according to $[t]^{1 / 2}$ for (PAR30-FITC/HA) ${ }_{24}$ multilayers, built with HA of molecular weights of 29 (black) or $150 \mathrm{kDa}$ (red). $t=$ 0 corresponds to the end of the photobleaching step. 
upon the entropy associated with the PAR/HA complexation but rather upon the strength of the arginine/HA monomer interaction and thus to the reaction heat. This suggests some correlations between the strength of the interaction between the polyelectrolytes (i.e., reaction enthalpy), diffusion ability of one of them in the multilayer film, the exponential character of the film, and finally its antimicrobial properties.

All of the above data were obtained by using HA whose molecular weight is $150 \mathrm{kDa}$. We also investigated if the molecular weight of HA has an influence on the antimicrobial properties. For this purpose, we performed antimicrobial experiments with (PAR30/HA $)_{24}$ films using $\mathrm{HA}$ chains with a molecular weight of $29 \mathrm{kDa}$. The molecular weight of $\mathrm{HA}$ seems to have no influence on the antimicrobial character of the film; a similar bacterial growth inhibition was obtained for both HA $29 \mathrm{kDa}$ and HA $150 \mathrm{kDa}$ based films (Figure 4a,b). We also checked that the molecular weight of HA does not affect the diffusion ability of the PAR chains in the multilayer (Figure $4 \mathrm{c}$ ). This was expected since one PAR chain should interact with different HA chains in the multilayer. Moreover, ITC experiments show that the interaction of PAR with HA is endothermic, independently of the molecular weight of HA (Table 2 and Figure S6).

Table 2. Reaction Enthalpies for the Investigated PAR30/ HA Couple

\begin{tabular}{ccc}
$\begin{array}{c}\text { MW of HA } \\
(\mathrm{kDa})\end{array}$ & $\begin{array}{c}\text { antimicrobial properties of the } \\
(\mathrm{PAR} 30 / \mathrm{HA}) \text { film }\end{array}$ & $\begin{array}{c}\text { reaction enthalpy } \\
(\mathrm{kJ} \mathrm{mol})\end{array}$ \\
\hline 29 & + & +0.78 \\
150 & + & +0.35 \\
\hline
\end{tabular}

\section{CONCLUSION}

Effective contact-killing antimicrobial coatings constitute a potential remedy for some nosocomial infections related with medical devices. We demonstrated previously that (PAR30/ HA) films present a strong antimicrobial activity against bacteria known to be involved in nosocomial infections. Here we investigated the influence of the polyanion component on the physicochemical and bioactivity properties of the film. For this purpose, the antimicrobial property of multilayers based on PAR30 and a series of polyanions (four polysaccharides, one homopolypeptide, and one synthetic polymer) was studied. (PAR30/HA $)_{24}$ multilayers were the only one showing an antimicrobial activity correlated with the most pronounced exponential growth regime and the highest diffusion ability of the PAR30 chains in the film, compared to the other systems. Finally, HA is the only investigated polyanion for which the reaction enthalpy with PAR30 is endothermic. PAR30 have the weakest interaction with HA which probably contributes to the strong diffusion ability of its chains in PAR30/HA films. These results explain, at a molecular level, the diffusion ability of PAR30 and thus the origin of the contact-killing property of this film.

\section{ASSOCIATED CONTENT}

\section{S Supporting Information}

The Supporting Information is available free of charge on the ACS Publications website

Evolution of thicknesses as a function of the number of adsorbed layers and estimated from QCM-D experiments, observation by confocal microscopy of the $(x, z)$ sections, and isothermal titration calorimetry data

\section{AUTHOR INFORMATION}

\section{Corresponding Authors}

*(P.L.) E-mail: philippe.lavalle@inserm.fr.

*(P.S.) E-mail: pierre.schaaf@inserm.fr.

ORCID

Angela Mutschler: 0000-0002-8226-977X

Nihal Engin Vrana: 0000-0002-5398-6710

Fouzia Boulmedais: 0000-0002-4934-9276

Philippe Lavalle: 0000-0001-8798-912X

Notes

The authors declare no competing financial interest.

\section{ACKNOWLEDGMENTS}

This project has received funding from the European Union's Seventh Framework Programme for research, technological development, and demonstration under Grant Agreement No. 602694 (IMMODGEL). We thank K. Benmlih and C. Bouthier for their support.

\section{ABBREVIATIONS}

ALG, alginate; CSA, chondroitin sulfate A; FRAP, fluorescence recovery after photobleaching; HA, hyaluronic acid; HEP, heparin; ITC, isothermal titration calorimetry; PAR, poly(arginine); PGA, poly(L-glutamic acid); PSS, poly(styrenesulfonate); QCM-D, quartz crystal microbalance dissipation; TRIS, 2-amino-2-hydroxyméthyl-1,3-propanediol

\section{REFERENCES}

(1) Sampedro, M. F.; Patel, R. Infections associated with long-term prosthetic devices. Infect. Dis. Clin. North Am. 2007, 21, 785-819.

(2) Schierholz, J. M.; Beuth, J. Implant infections: a haven for opportunistic bacteria. J. Hosp. Infect. 2001, 49, 87-93.

(3) Gbejuade, H. O.; Lovering, A. M.; Webb, J. C. The role of microbial biofilms in prosthetic joint infections. Acta Orthop. 2015, 86, 147-158.

(4) Campoccia, D.; Montanaro, L.; Arciola, C. R. The significance of infection related to orthopedic devices and issues of antibiotic resistance. Biomaterials 2006, 27, 2331-2339.

(5) Campoccia, D.; Montanaro, L.; Arciola, C. R. A review of the biomaterials technologies for infection-resistant surfaces. Biomaterials 2013, 34, 8533-8554.

(6) Decher, G. Fuzzy nanoassemblies: toward layered polymeric multicomposites. Science 1997, 277, 1232-1237.

(7) Tang, Z. Y.; Wang, Y.; Podsiadlo, P.; Kotov, N. A. Biomedical applications of layer-by-layer assembly: from biomimetics to tissue engineering. Adv. Mater. 2006, 18, 3203-3224.

(8) Richardson, J. J.; Cui, J.; Bjornmalm, M.; Braunger, J. A.; Ejima, H.; Caruso, F. Innovation in layer-by-layer assembly. Chem. Rev. 2016, $116,14828-14867$.

(9) Wang, B. L.; Ren, K. F.; Chang, H.; Wang, J. L.; Ji, J. Construction of degradable multilayer films for enhanced antibacterial properties. ACS Appl. Mater. Interfaces 2013, 5, 4136-4143.

(10) Shukla, A.; Fleming, K. E.; Chuang, H. F.; Chau, T. M.; Loose, C. R.; Stephanopoulos, G. N.; Hammond, P. T. Controlling the release of peptide antimicrobial agents from surfaces. Biomaterials 2010, 31, 2348-57.

(11) Séon, L.; Lavalle, P.; Schaaf, P.; Boulmedais, F. Polyelectrolyte multilayers: a versatile tool for preparing antimicrobial coatings. Langmuir 2015, 31, 12856-12872.

(12) Lichter, J. A.; Thompson, M. T.; Delgadillo, M.; Nishikawa, T.; Rubner, M. F.; Van Vliet, K. J. Substrata mechanical stiffness can regulate adhesion of viable bacteria. Biomacromolecules 2008, 9, 15711578.

(13) Cado, G.; Aslam, R.; Séon, L.; Garnier, T.; Fabre, R.; Parat, A.; Chassepot, A.; Voegel, J.-C.; Senger, B.; Schneider, F.; Frère, Y.; Jierry, 
L.; Schaaf, P.; Kerdjoudj, H.; Metz-Boutigue, M.-H.; Boulmedais, F. Self-defensive biomaterial coating against bacteria and yeasts: Polysaccharide multilayer film with embedded antimicrobial peptide. Adv. Funct. Mater. 2013, 23, 4801-4809.

(14) Min, J.; Braatz, R. D.; Hammond, P. T. Tunable staged release of therapeutics from layer-by-layer coatings with clay interlayer barrier. Biomaterials 2014, 35, 2507-2517.

(15) Podsiadlo, P.; Paternel, S.; Rouillard, J. M.; Zhang, Z. F.; Lee, J.; Lee, J. W.; Gulari, L.; Kotov, N. A. Layer-by-layer assembly of nacrelike nanostructured composites with antimicrobial properties. Langmuir 2005, 21, 11915-11921.

(16) Agarwal, A.; Nelson, T. B.; Kierski, P. R.; Schurr, M. J.; Murphy, C. J.; Czuprynski, C. J.; McAnulty, J. F.; Abbott, N. L. Polymeric multilayers that localize the release of chlorhexidine from biologic wound dressings. Biomaterials 2012, 33, 6783-6792.

(17) Özçelik, H.; Vrana, N. E.; Gudima, A.; Riabov, V.; Gratchev, A.; Haikel, Y.; Metz-Boutigue, M. H.; Carrado, A.; Faerber, J.; Roland, T.; Kluter, H.; Kzhyshkowska, J.; Schaaf, P.; Lavalle, P. Harnessing the multifunctionality in nature: a bioactive agent release system with selfantimicrobial and immunomodulatory properties. Adv. Healthcare Mater. 2015, 4, 2026-2036.

(18) Mutschler, A.; Tallet, L.; Rabineau, M.; Dollinger, C.; MetzBoutigue, M.-H.; Schneider, F.; Senger, B.; Vrana, N. E.; Schaaf, P.; Lavalle, P. Unexpected bactericidal activity of poly(arginine)/ hyaluronan nanolayered coatings. Chem. Mater. 2016, 28, 8700-8709.

(19) Picart, C.; Mutterer, J.; Richert, L.; Luo, Y.; Prestwich, G. D.; Schaaf, P.; Voegel, J.-C.; Lavalle, P. Molecular basis for the explanation of the exponential growth of polyelectrolyte multilayers. Proc. Natl. Acad. Sci. U. S. A. 2002, 99, 12531-12535.

(20) Sauerbrey, G. Use of quartz crystal vibrator for weighting thin films on a microbalance. Eur. Phys. J. A 1959, 155, 206-222.

(21) Voinova, M. V.; Rodahl, M.; Jonson, M.; Kasemo, B. Viscoelastic acoustic response of layered polymer films at fluid-solid interfaces: continuum mechanics approach. Phys. Scr. 1999, 59, 391-396.

(22) Soumpasis, D. M. Theoretical-analysis of fluorescence photobleaching recovery experiments. Biophys. J. 1983, 41, 95-97.

(23) Picart, C.; Mutterer, J.; Arntz, Y.; Voegel, J. C.; Schaaf, P.; Senger, B. Application of fluorescence recovery after photobleaching to diffusion of a polyelectrolyte in a multilayer film. Microsc. Res. Tech. 2005, 66, 43-57.

(24) Crouzier, T.; Picart, C. Ion pairing and hydration in polyelectrolyte multilayer films containing polysaccharides. Biomacromolecules 2009, 10, 433-442.

(25) Guillaume-Gentil, O.; Zahn, R.; Lindhoud, S.; Graf, N.; Voros, J.; Zambelli, T. From nanodroplets to continuous films: how the morphology of polyelectrolyte multilayers depends on the dielectric permittivity and the surface charge of the supporting substrate. Soft Matter 2011, 7, 3861-3871.

(26) Steinberg, J. P.; Clark, C. C.; Hackman, B. O. Nosocomial and community-acquired Staphylococcus aureus bacteremias from 1980 to 1993: impact of intravascular devices and methicillin resistance. Clin. Infect. Dis. 1996, 23, 255-259.

(27) Laugel, N.; Betscha, C.; Winterhalter, M.; Voegel, J. C.; Schaaf, P.; Ball, V. Relationship between the growth regime of polyelectrolyte multilayers and the polyanion/polycation complexation enthalpy. $J$. Phys. Chem. B 2006, 110, 19443-19449. 